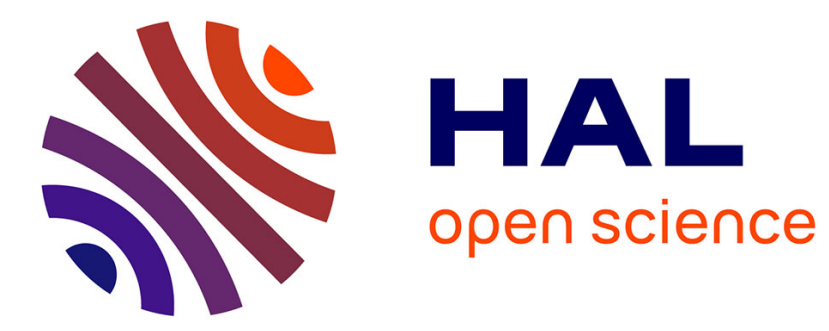

\title{
Dynamics of Multistage Bladed Disks Systems
}

Denis Laxalde, Jean-Pierre Lombard, Fabrice Thouverez

\section{To cite this version:}

Denis Laxalde, Jean-Pierre Lombard, Fabrice Thouverez. Dynamics of Multistage Bladed Disks Systems. Journal of Engineering for Gas Turbines and Power, 2007, 129 (4), pp.1058-1064. 10.1115/1.2747641 . hal-00243153

\section{HAL Id: hal-00243153 \\ https://hal.science/hal-00243153}

Submitted on 16 Mar 2009

HAL is a multi-disciplinary open access archive for the deposit and dissemination of scientific research documents, whether they are published or not. The documents may come from teaching and research institutions in France or abroad, or from public or private research centers.
L'archive ouverte pluridisciplinaire HAL, est destinée au dépôt et à la diffusion de documents scientifiques de niveau recherche, publiés ou non, émanant des établissements d'enseignement et de recherche français ou étrangers, des laboratoires publics ou privés. 


\title{
Dynamics of Multi-Stage Bladed Disks Systems
}

\author{
Denis Laxalde ${ }^{(\mathrm{a}, \mathrm{b})}$, Jean-Pierre Lombard ${ }^{(\mathrm{b})}$ and Fabrice Thouverez ${ }^{(\mathrm{a})}$ \\ (a) Laboratoire de Tribologie et Dynamique des Systèmes UMR-CNRS 5513, \\ École Centrale de Lyon, 36 avenue Guy de Collongue, 69134 Ecully Cedex, France \\ ${ }^{(b)}$ Snecma - Safran group \\ 77550 Moissy-Cramayel, France
}

\begin{abstract}
This paper presents a new and original method for dynamical analysis of multi-stage cyclic structures such as turbomachinery compressors or turbines. Each stage is modeled cyclically by its elementary sector and the inter-stage coupling is achieved through a cyclic recombination of the interface degrees of freedom. This method is quite simple to set-up; it allows to handle the finite element models of each stage's sector directly and, as in classical cyclic symmetry analysis, to study the nodal diameter problems separately. The method is first validated on a simple case study which shows good agreements with a complete 360 degrees reference calculation. An industrial example involving two HP compressor stages is then presented. Then the forced response application is presented in which synchronous of engine order type excitations are considered.
\end{abstract}

\section{Introduction}

The mechanical design of gas turbine rotors is traditionally fulfilled through stage by stage analysis. However, in modern engines, dynamical phenomena involving multiple stages (adjoining or not) of bladed disks tend to appear and can then be of primary interest for gas turbine professionals. On the other hand, the traditional (single stage) analysis of bladed disks dynamics is usually performed using the cyclic symmetry reduction which allows to analyze a complete rotationally periodic structure by considering only one of its elementary sector. Examples in the literature are numerous on this subject, from the early developments of cyclic symmetry method Thomas (1979); Wildheim (1981) to specific researches on bladed disks dynamics Srinivasan (1984); Slater (1999). Withal, in such analysis, the interstage coupling is necessarily neglected, its eventual effects can not be accounted for and this can lead to significant deviations from the real (multi-stage) system. For example, Bladh et al. Bladh et al. (2003) have show that the interstage coupling can significantly affect the dynamics of the multistage assembly and that the traditional single stage analysis can, in some cases, leads to an underestimation of vibratory levels.

Few strategies exist to answer the challenge of multistage modeling and a major issue is that, since a multistage assembly of cyclic structures is a priori no longer cyclic, the cyclic symmetry reduction cannot be used straightforward. Some Rzadkowski and Drewczynski (2006) have used full 360 degrees models to study the (free and forced) dynamics of multi-stage systems. However, as such techniques often yield prohibitive computational costs. Component mode synthesis methods (with multilevel reductions) has also been used Bladh et al. (2001); Song et al. (2005) but these strategies can be somewhat difficult to use in a design process.

In the present paper, we put forward a new strategy to study the dynamics of multistage assemblies using a cyclic symmetry reduction. This method handles the sector finite element 
modeling directly and involves a realistic interstage coupling based on a cyclic recombination of the interface on the chosen nodal diameter. Following a brief review of the cyclic symmetry method (section 2), the proposed method will be detailed in section 3. Then, some numerical examples and results will be presented in section 4 to validate the method and highlight the importance of such multi-stage calculations in the design of turbomachinery components.

\section{Cyclic symmetry for single stages}

In this first section, a brief review of the classical cyclic symmetry modeling is done which mainly aims at defining some notations. A cyclic structure composed of $N$ sectors is considered. Its displacement vector can be split as,

$$
\boldsymbol{x}=\left[\boldsymbol{x}_{0}, \boldsymbol{x}_{1}, \ldots, \boldsymbol{x}_{N-1}\right]^{T}
$$

where $\boldsymbol{x}_{j}$ is the displacement vector of the $j$-th sector.

As a consequence of the rotational periodicity, the mode shapes are such that each sector has the same deflection and a constant phase difference from the adjacent sectors. This inter-sector phase angle is $\beta_{n}=2 \pi n / N$ and the harmonic index $n=0,1, \ldots, N-1$ is called the nodal diameter number.

As a result, any dynamical problem on the complete cyclic structure,

$$
M \ddot{\boldsymbol{x}}+C \dot{\boldsymbol{x}}+K \boldsymbol{x}=\boldsymbol{f}_{e}
$$

can be transform (exactly) into reduced problems on the reference sector (corresponding to each nodal diameter number $n$ )

$$
M_{n} \ddot{\tilde{\boldsymbol{x}}}_{n}+C_{n} \dot{\tilde{\boldsymbol{x}}}_{n}+K_{n} \tilde{\boldsymbol{x}}_{n}=\tilde{\boldsymbol{f}}_{e, n}
$$

The displacement vector, in the cyclic base, $\tilde{\boldsymbol{x}}_{n}$ is obtained from the reference sector's displacement vector in which the left inter-sector degrees-of-freedom, ${ }^{l} \boldsymbol{x}_{0}$, have been eliminated using the relation to the right inter-sector degrees-of-freedom, ${ }^{r} \boldsymbol{x}_{0}$, for each $n$ nodal diameter,

$$
{ }^{l} \boldsymbol{x}_{0}=e^{-\frac{2 i \pi n}{N} r} \boldsymbol{x}_{0}
$$

The reduced matrices $M_{n}, C_{n}$ and $K_{n}$ are obtained by applied this periodicity conditions on the reference sector's structural matrices.

As the considered structures have symmetric structural matrices, $\tilde{\boldsymbol{x}}_{n}$ and $\tilde{\boldsymbol{x}}_{N-n}$ contains the same relevant information and thus among the $N$ possible values of the nodal diameter index $n$ only $N_{s}$ of these are to be calculated,

$$
N_{s}= \begin{cases}N / 2+1 & \text { if } N \text { is even } \\ (N+1) / 2 & \text { if } N \text { is odd }\end{cases}
$$

Each reduced problem (3) is solved in cyclic coordinates $\tilde{\boldsymbol{x}}_{n}$ (for the reference sector) and the transformation in physical coordinates (complete structure) is given by:

$$
\boldsymbol{x}=\boldsymbol{e}_{n} \otimes \tilde{\boldsymbol{x}}_{n} \text { with } \boldsymbol{e}_{n}=\left[\begin{array}{lllll}
1 & e^{\frac{2 j \pi n}{N}} & e^{\frac{4 j \pi n}{N}} & \ldots & e^{\frac{2(N-1) j \pi n}{N}}
\end{array}\right]^{T}
$$

where $\otimes$ is the Kronecker product.

For further readings, see references Thomas (1979); Wildheim (1981); Henry (1980). 


\section{$3 \quad$ A multistage cyclic symmetry method}

We suggest a new strategy to analyze the dynamics of multistage cyclic structures. This method was originally proposed by the authors in Laxalde et al. (2007a). It is called multistage cyclic symmetry since the cyclic symmetry strategy is used to model each stage. The interstage coupling is ensured through a cyclic expansion of the interface degrees of freedom of each stages. The method will be detailed based on the example of two stages respectively composed of $N_{1}$ and $N_{2}$ sectors $\left(N_{1}<N_{2}\right)$. This method is applicable for any dynamical problems in free and forced responses. In this paper, we focused on linear applications for modal analysis and forced steady-state response. Extension to non-linear steady-state response calculations can easily be considered by combining cyclic symmetry approach with Fourier methods Petrov (2004); Laxalde et al. (2007b).

Equations of the motion. We consider the general dynamical problem involving several coupled stages, each having a second-order equation of the motion of the form,

$$
M^{(s)} \ddot{\boldsymbol{x}}^{(s)}+C^{(s)} \dot{\boldsymbol{x}}^{(s)}+K^{(s)} \boldsymbol{x}^{(s)}=\boldsymbol{f}_{e}^{(s)} \quad(s=1 \text { or } 2)
$$

in which, $M^{(s)}, C^{(s)}$ and $K^{(s)}$ are mass, damping and stiffness matrices, $\boldsymbol{x}^{(s)}$ is the displacement vector and $\boldsymbol{f}_{e}^{(s)}$ is the external forces on the full stage $s$.

Since each stage is rotationally periodic, it can be represented by its elementary sector in its own cyclic base by the following uncoupled nodal diameters equations,

$$
M_{n}^{(s)} \ddot{\tilde{\boldsymbol{x}}}_{n}^{(s)}+C_{n}^{(s)} \dot{\tilde{\boldsymbol{x}}}_{n}^{(s)}+K_{n}^{(s)} \tilde{\boldsymbol{x}}_{n}^{(s)}=\tilde{\boldsymbol{f}}_{e, n}^{(s)}, \text { for } n \in\left[0 \ldots N_{s}^{(s)}-1\right]
$$

The proposed method aims at coupling the models of each stage in their own cyclic components.

Interstage coupling. At the interface between stages 1 and 2, the coupling is achieved by enforcing continuity of displacements (or other variables) on the interstage boundary in the common physical base,

$$
\Delta^{b} \boldsymbol{x}=A^{b} \boldsymbol{x}^{(1)}-{ }^{b} \boldsymbol{x}^{(2)}=\mathbf{0}
$$

where the left superscript ${ }^{b}$. refers the restrictions to the interstage boundary degrees-of-freedom and $A$ is a constraint matrix which makes the two interstage meshes compatible (multi-point constraints).

Equation. (9) can be rewritten, using each stage's cyclic base, as,

$$
A \sum_{n=0}^{N_{1}-1} \boldsymbol{e}_{N_{1}, n} \otimes{ }^{b} \tilde{\boldsymbol{x}}_{i, n}^{(1)}+\sum_{p=0}^{N_{2}-1} \boldsymbol{e}_{N_{2}, p} \otimes{ }^{b} \tilde{\boldsymbol{x}}_{i, p}^{(2)}=0
$$

Then, we can project equation (10) on any nodal diameter vector of stage 2 cyclic base ( $p \in$ $\left.\left[0 \ldots N_{2}-1\right]\right)$ and obtain,

$$
\left(\boldsymbol{e}_{N_{2}, p}^{*} \otimes \mathcal{I}_{b_{2}}\right) A \sum_{n=0}^{N_{1}-1} \boldsymbol{e}_{N_{1}, n} \otimes{ }^{b} \tilde{\boldsymbol{x}}_{i, n}^{(1)}+{ }^{b} \tilde{\boldsymbol{x}}_{i, p}^{(2)}=0
$$

where the identity mtrices $\mathcal{I}_{b_{s}}(s=1,2)$ ensure the compatibility of the interstage interface.

In equation (11), the coupling terms between nodal diameters $n$ and $p$ of each stage's base are

$$
\mathcal{C}(p, n)=\left(\boldsymbol{e}_{N_{2}, p}^{*} \otimes \mathcal{I}_{b_{2}}\right) A\left(\boldsymbol{e}_{N_{1}, n} \otimes \mathcal{I}_{b_{1}}\right)
$$


The cornerstone of the proposed method is to neglect the coupling between "distinct" nodal diameters of each stage's base. We can, here, define the correspondence of nodal diameters indexes between the two stage's bases (which may not be obvious since the stages have different number of sectors). For any nodal diameter index $p \in\left[0 \ldots N_{2}\right]$ of stage 2 , we can define the corresponding "equivalent" nodal diameter index of stage $1 n(p)$ as,

$$
n(p)= \begin{cases}p & \text { if } p \leq N_{1} \\ N_{1}- \begin{cases}p\left(\bmod N_{1}\right) & \text { if } N_{1} \text { is odd } \\ p\left(\bmod N_{1}\right) & \text { if } N_{1} \text { is even }\end{cases} & \text { if } p>N_{1}\end{cases}
$$

This correspond to the aliasing of the discrete Fourier transform. The "nodal diameter equivalence" is illustrated by figures (1), with the even (fig. 1(a)) and odd (fig. 1(b)) cases. Finally, the

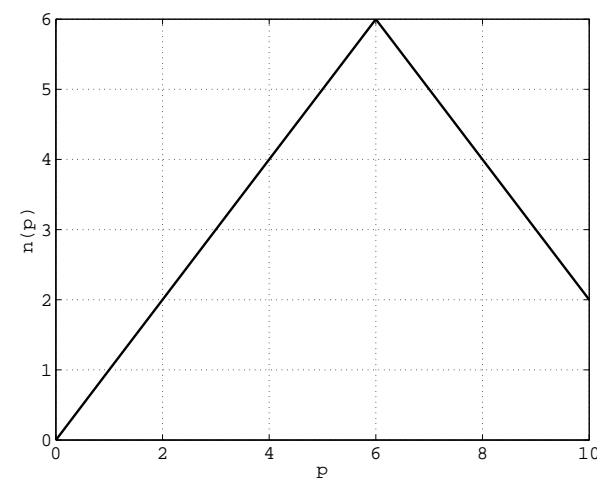

(a) $N_{1}=12$ and $N_{2}=20$

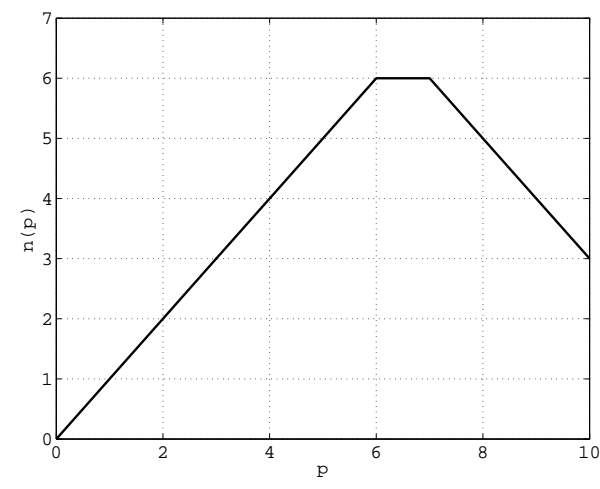

(b) $N_{1}=13$ and $N_{2}=20$

Figure 1. "Nodal diameter equivalence" rule

restriction of the inter-stage projection given by equation (11) to "equivalent" nodal diameters leads to:

$$
\left(\boldsymbol{e}_{N_{2}, p}^{*} \otimes \mathcal{I}_{b_{2}}\right) A\left(\boldsymbol{e}_{N_{1}, n(p)} \otimes \mathcal{I}_{b_{1}}\right)^{b} \tilde{\boldsymbol{x}}_{i, n(p)}^{(1)}+{ }^{b} \tilde{\boldsymbol{x}}_{i, p}^{(2)}=0
$$

This assumption will next be referred as the one-to-one nodal diameter coupling assumption.

Multistage cyclic symmetry reduction. The one-to-one coupling assumption makes possible the multi-stage coupling in the cyclic bases for each nodal diameters separately. We can append two stages models in their own cyclic base and according to the previously defined nodal diameter correspondence relation (13) as,

$$
\left(\begin{array}{cc}
M_{n(p)}^{(1)} & 0 \\
0 & M_{p}^{(2)}
\end{array}\right)\left[\begin{array}{c}
\ddot{\boldsymbol{x}}_{n(p)}^{(1)} \\
\ddot{\tilde{\boldsymbol{x}}}_{p}^{(2)}
\end{array}\right]+\left(\begin{array}{cc}
C_{n(p)}^{(1)} & 0 \\
0 & C_{p}^{(2)}
\end{array}\right)\left[\begin{array}{c}
\dot{\tilde{\boldsymbol{x}}}_{n(p)}^{(1)} \\
\dot{\tilde{\boldsymbol{x}}}_{p}^{(2)}
\end{array}\right]+\left(\begin{array}{cc}
K_{n(p)}^{(1)} & 0 \\
0 & K_{p}^{(2)}
\end{array}\right)\left[\begin{array}{c}
\tilde{\boldsymbol{x}}_{n(p)}^{(1)} \\
\tilde{\boldsymbol{x}}_{p}^{(2)}
\end{array}\right]=\left[\begin{array}{c}
\tilde{\boldsymbol{f}}_{e, n(p)}^{(1)} \\
\tilde{\boldsymbol{f}}_{e, p}^{(2)}
\end{array}\right]
$$

Next, if each stage displacements vector is split as,

$$
\tilde{\boldsymbol{x}}_{n(p)}^{(1)}=\left[{ }^{b} \tilde{\boldsymbol{x}}_{n(p)}^{(1)},{ }^{i} \tilde{\boldsymbol{x}}_{n(p)}^{(1)}\right]^{T} \text { and } \tilde{\boldsymbol{x}}_{p}^{(2)}=\left[{ }^{b} \tilde{\boldsymbol{x}}_{p}^{(2)},{ }^{i} \tilde{\boldsymbol{x}}_{p}^{(2)}\right]^{T}
$$

Eq. (14) can be used to eliminate the cyclic components of the interstage boundary of stage 2,

$$
\left[\begin{array}{c}
\tilde{\boldsymbol{x}}_{n(p)}^{(1)} \\
\tilde{\boldsymbol{x}}_{p}^{(2)}
\end{array}\right]=\mathcal{T}_{p} \tilde{\boldsymbol{x}}_{p}^{(1,2)}
$$


with,

$$
\mathcal{T}_{p}=\left(\begin{array}{ccc}
\mathcal{I}_{b_{1}} & 0 & 0 \\
0 & \mathcal{I}_{i_{1}} & 0 \\
\mathcal{B}_{p} & 0 & 0 \\
0 & 0 & \mathcal{I}_{i_{2}}
\end{array}\right), \mathcal{B}_{p}=\left(\boldsymbol{e}_{N_{2}, p}^{*} \otimes \mathcal{I}_{b_{2}}\right) A\left(\boldsymbol{e}_{N_{1}, n(p)} \otimes \mathcal{I}_{b_{1}}\right)
$$

and,

$$
\tilde{\boldsymbol{x}}_{p}^{(1,2)}=\left[\begin{array}{lll}
b \tilde{\boldsymbol{x}}_{n(p)}^{(1)} & i \tilde{\boldsymbol{x}}_{n(p)}^{(2)} & i \tilde{\boldsymbol{x}}_{p}^{(2)}
\end{array}\right]^{T}
$$

The final multi-stage one-to-one nodal diameter dynamical problem can be defined as:

$$
M_{p}^{(1,2)} \ddot{\tilde{\boldsymbol{x}}}_{p}^{(1,2)}+C_{p}^{(1,2)} \dot{\tilde{\boldsymbol{x}}}_{p}^{(1,2)}+K_{p}^{(1,2)} \tilde{\boldsymbol{x}}_{p}^{(1,2)}=\tilde{\boldsymbol{f}}_{e, p}^{(1,2)}
$$

with,

$$
\begin{aligned}
M_{p}^{(1,2)} & =\mathcal{T}_{p}^{T}\left(\begin{array}{cc}
M_{n(p)}^{(1)} & 0 \\
0 & M_{p}^{(2)}
\end{array}\right) \mathcal{T}_{p} \\
C_{p}^{(1,2)} & =\mathcal{T}_{p}^{T}\left(\begin{array}{cc}
C_{n(p)}^{(1)} & 0 \\
0 & C_{p}^{(2)}
\end{array}\right) \mathcal{T}_{p} \\
K_{p}^{(1,2)} & =\mathcal{T}_{p}^{T}\left(\begin{array}{cc}
K_{n(p)}^{(1)} & 0 \\
0 & K_{p}^{(2)}
\end{array}\right) \mathcal{T}_{p}
\end{aligned}
$$

This method allows the study of nodal diameter modes separately as for individual stages, each being modeled with its own elementary sector. The size of problems (15) or (17) is close to the sum of the two individual stages problem sizes. The accuracy of this method in modal analysis and particularly the validity of the one-to-one nodal diameter coupling will be demonstrated by numerical examples in section 4.1 .

Superposition. The multistage cyclic symmetry formalism allows to isolate the nodal diameters contributions of the response. However, in some cases and particularly in forced response, the dynamics of the multi-stage system can feature simultaneous contributions of different nodal diameters. To handle with such problems, two strategies can be used. The first is to use the "superposition principle" as in typical single stage cyclic symmetry analysis; that is to say, the external forcing distribution on each stage is first decomposed into elementary cyclic forces corresponding to nodal diameter contributions of each stage and then each uncoupled, nodal diameter projected, equation can be solved individually. The global response is then obtained by summing all contributions. The second is to consider all harmonic components of the excitation (or response) in the projection step of the multi-stage cyclic symmetry method. Example given, if two harmonics (or nodal diameter numbers) $p$ and $q$ are considered, the projection equation (14) becomes,

$$
\mathcal{B}_{p, q}\left[\begin{array}{l}
\tilde{\boldsymbol{x}}_{i, n(p)}^{(1)} \\
\tilde{\boldsymbol{x}}_{i, n(q)}^{(1)}
\end{array}\right]+\left[\begin{array}{l}
\tilde{\boldsymbol{x}}_{i, p}^{(2)} \\
\tilde{\boldsymbol{x}}_{i, q}^{(2)}
\end{array}\right]=0
$$

with

$$
\mathcal{B}_{p, q}=\left(\left[\boldsymbol{e}_{N_{2}, p}, \boldsymbol{e}_{N_{2}, q}\right]^{*} \otimes \mathcal{I}_{b_{2}}\right) A\left(\left[\boldsymbol{e}_{N_{1}, n(p)}, \boldsymbol{e}_{N_{1}, n(q)}\right] \otimes \mathcal{I}_{b_{1}}\right)
$$




\section{Numerical results}

The next of the paper is dedicated to numerical applications. First, a validation example will be used to evaluate the performances of the proposed method in modal analysis. Then, an industrial application based on two HP compressor rotating stages will be presented in both modal and forced responses. The importance of multi-stage dynamic analysis will also be discussed.

\subsection{Validation example}

In this part we evaluate the performance of the Multistage cyclic symmetry method on a numerical example. We consider two stages of cyclic structures with simple geometry; the first being composed of 24 elementary sectors and the second of 45 elementary sectors. The figure 2 shows the finite elements meshes of the two elementary sectors and of the whole structure. The results
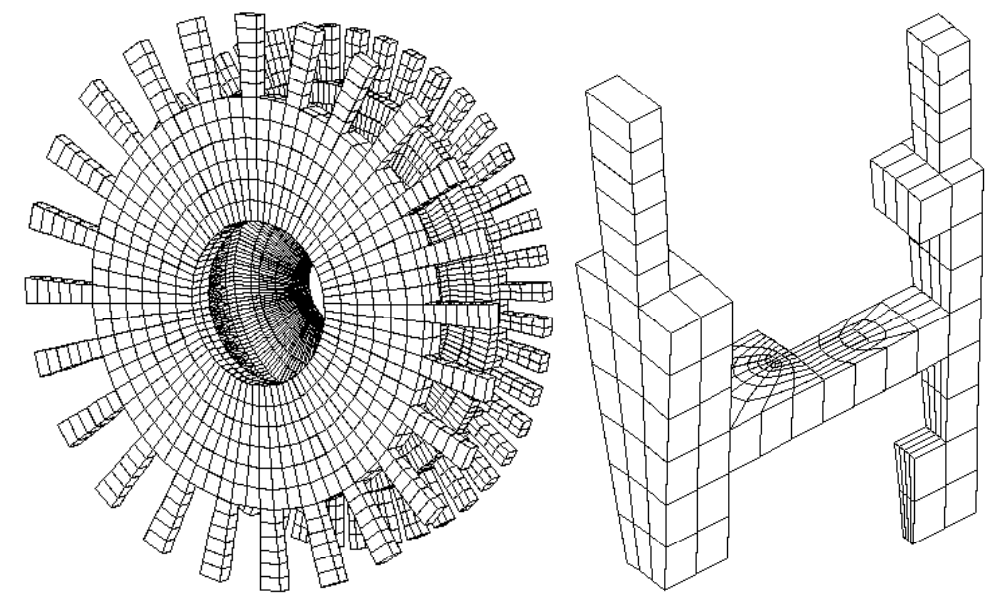

Figure 2. Finite element model of two cyclic structures $-N_{1}=24, N_{2}=45$

on the Multistage cyclic symmetry method are compared to a full 360 degrees analysis taken as a reference.

Correlation on modal data Figure 3 gathers the eigenfrequencies calculated by the two methods plotted versus their nodal diameter index. The correlation appears to be correct. This attests for the quality of the prediction of the multistage cyclic symmetry method and the validity of the one-to-one nodal diameter projection assumption.

Next, we show and compare (in a qualitative way) the modal deformed shapes. Figures 4 and 5 show results from (a) the reference analysis and (b) the Multistage cyclic symmetry for various nodal diameter numbers. In these examples we can distinguish some modes localized in only one stage (figure 5) and some others with a clear multistage behavior (figure 4). Here again, the correlation is correct.

Correlation on frequency responses We now investigated the forced response of the two stages model of figure 2. We consider two distributions of forces on each blade of each stage with a spatial periodicity of $n$ ( $n$ nodal diameters), such that the force of the $j$-th blade of stage $s$ is,

$$
f_{j}^{(s)}=F_{0} \cos \frac{2 n \pi}{N_{s}}(j-1) \cos \omega t, s=1 \text { or } 2
$$




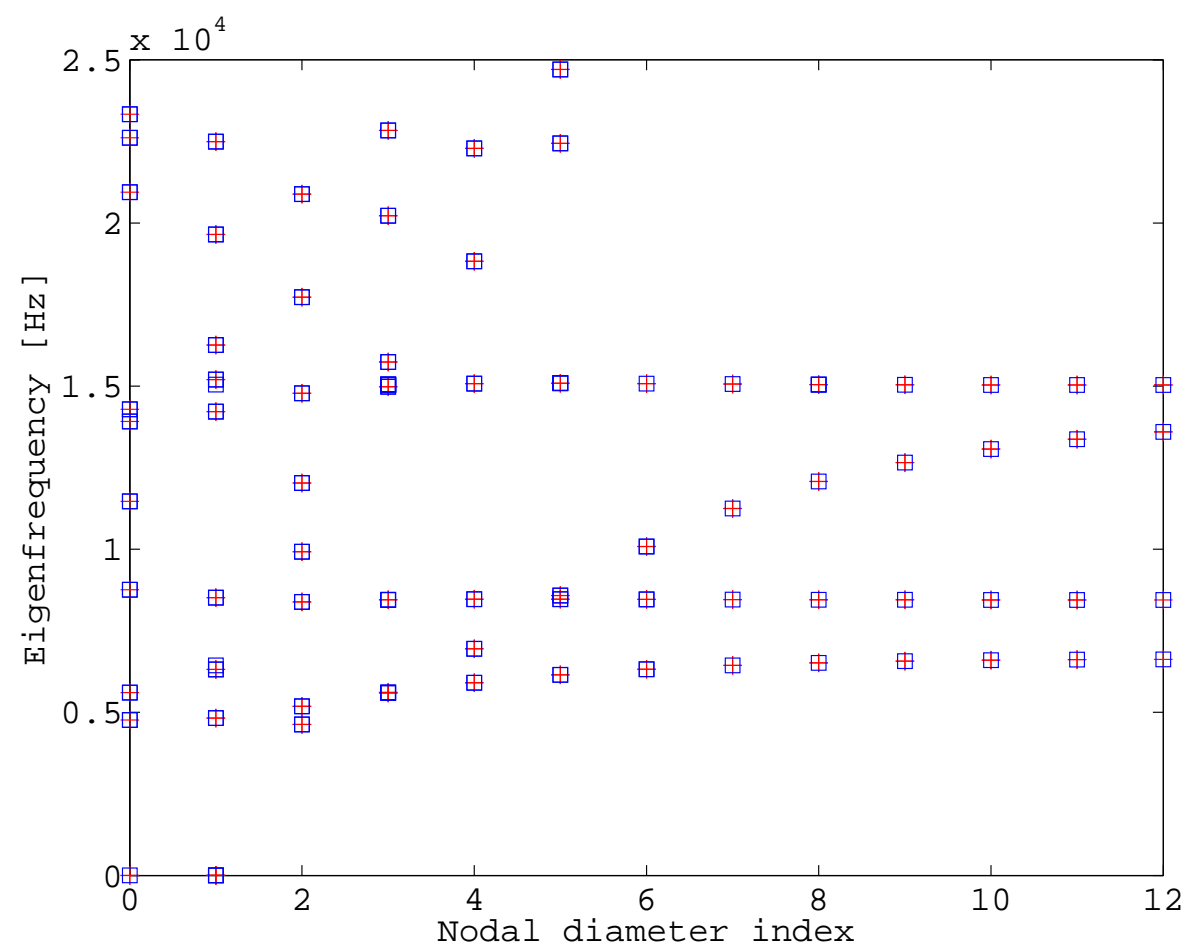

Figure 3. Eigenfrequency correlation, reference $\square /$ Multistage cyclic symmetry + .

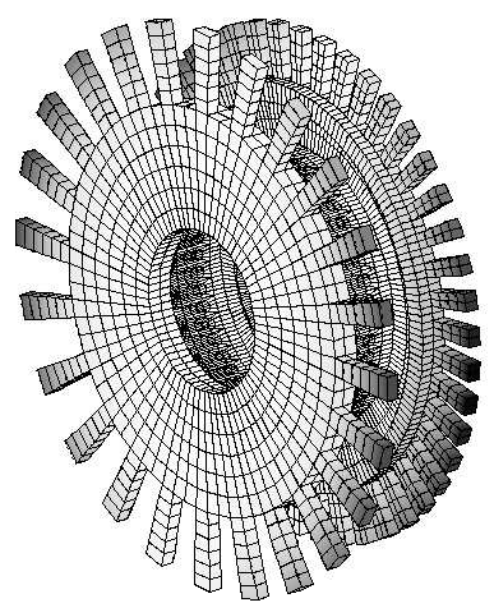

(a)

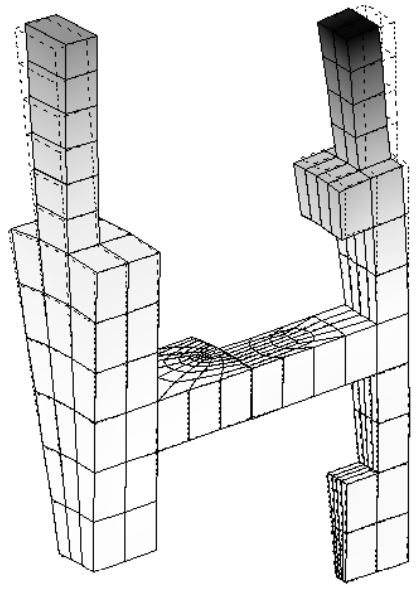

(b)

Figure 4. One nodal diameter mode at $4824 \mathrm{~Hz}$ 


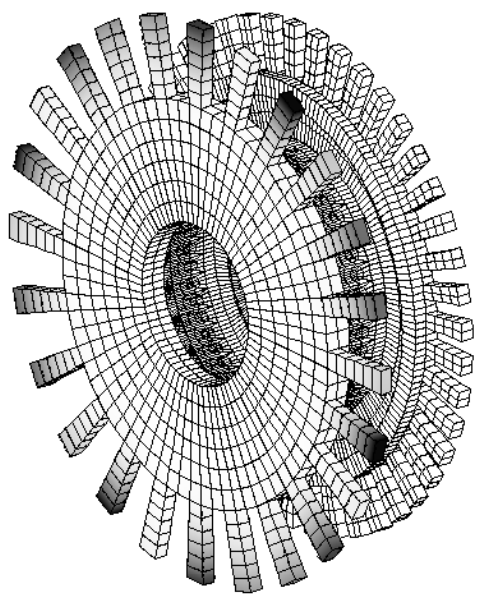

(a)

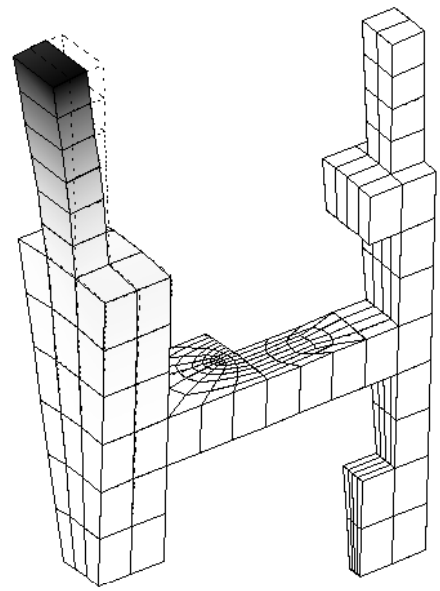

(b)

Figure 5. Five nodal diameter mode at $6147 \mathrm{~Hz}$

For a wave number of $n=2$, the frequency responses are displayed in figures 6 for the first blade of each stage. The correlation appears to be globally correct. The resonances (frequency and levels) are correctly represented by the multi-stage cyclic symmetry. There only appears small shifts on the antiresonances between the two calculations. This might be related to the one-to-one approximation of the multi-stage cyclic symmetry method described in section 3 . This is quite similar to a truncation effect in modal analysis. However, the global dynamics remains well approximated.

\subsection{Industrial case study}

Next, an industrial application of the multi-stage cyclic symmetry method is presented. Figures 7 show the finite element models (sectors) of two HP compressor blisks which respectively have 36 and 60 sectors. The computation times of a traditional multi-stage analysis (with 360 degrees models) are clearly prohibitive and thus useless during a realistic design process; in contrast, multi-stage cyclic symmetry analysis can provide satisfying results with reasonable computational times.

Modal analysis. For this representative model, we found interesting to show the influence of the interstage coupling by comparing the results of single stage analysis to the multistage case on a modal analysis. For the single stage analysis we applied a fixed boundary condition on the interstage interface. Figure 8 gathers the (normalized) eigenfrequencies of these three calculations. The influence of the interstage coupling is noticeable and new modes appear that could not be predicted by a single stage analysis. This is particularly observable in small nodal diameter numbers. In effect, in these cases, the blade/disk coupling is generally more important than for higher nodal diameter numbers where the disk's participation is usually smaller. In addition, we provide some example of modal deformed shapes from the multistage computation in figure 9. Some modes show a weak interstage coupling, such as the ones represented in figures 9(a) and 9(b), and feature nearly a single stage behavior. On the other hand, figure 9(c) displays a strongly (interstage) coupled behavior and can obviously not be represented in traditional single stage analysis. This comparison clearly shows the influence of the multistage coupling in the design of multi-stage blade disks systems. 

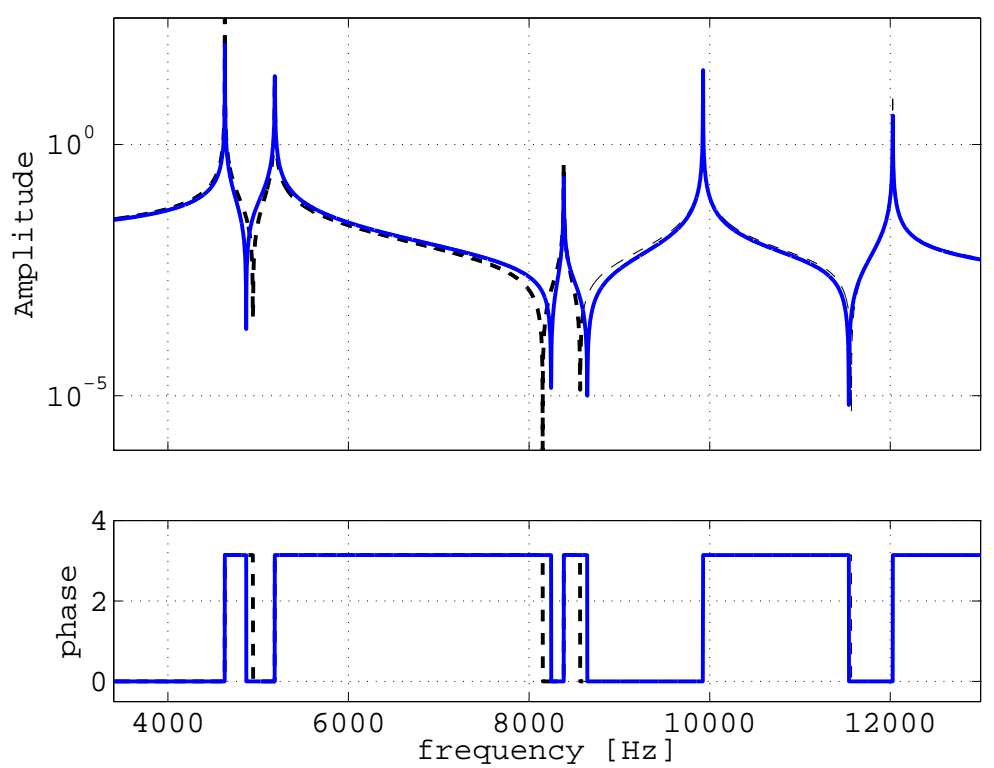

(a) Blade 1
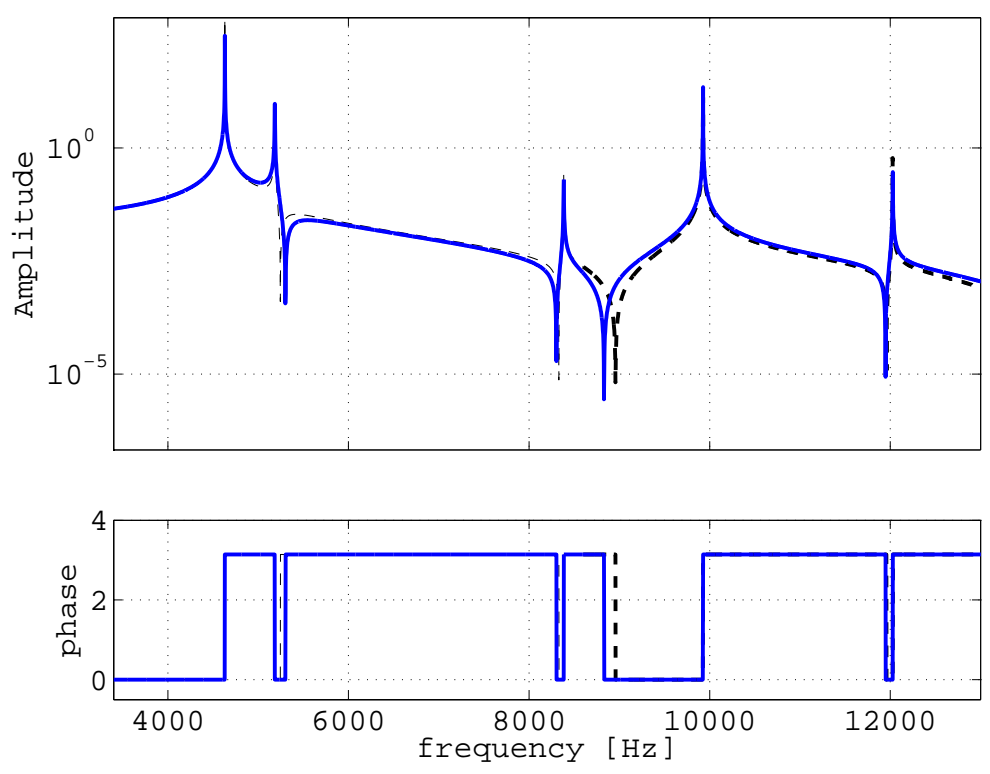

(b) Blade 2

Figure 6. FRF correlation; (- -) reference, (-) multi-stage cyclic symmetry 


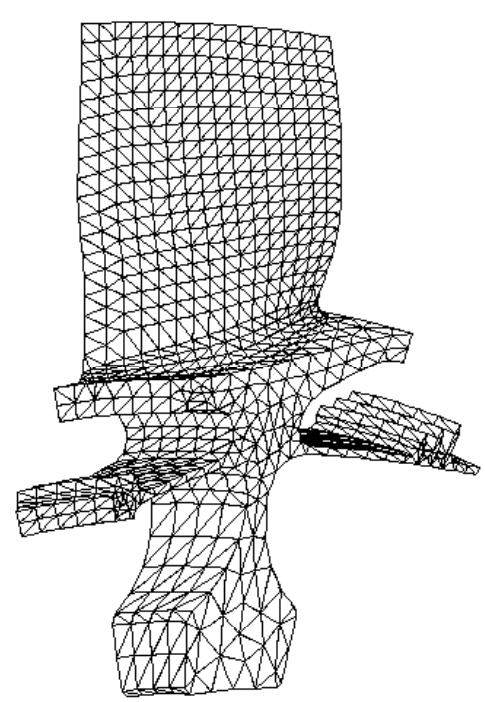

(a) $1^{\text {st }}$ stage -36 sectors

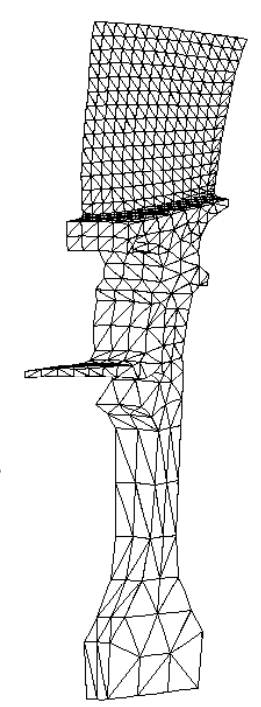

(b) $2^{\text {nd }}$ stage 60 sectors

Figure 7. Finite Element Models of two HP compressor blisks

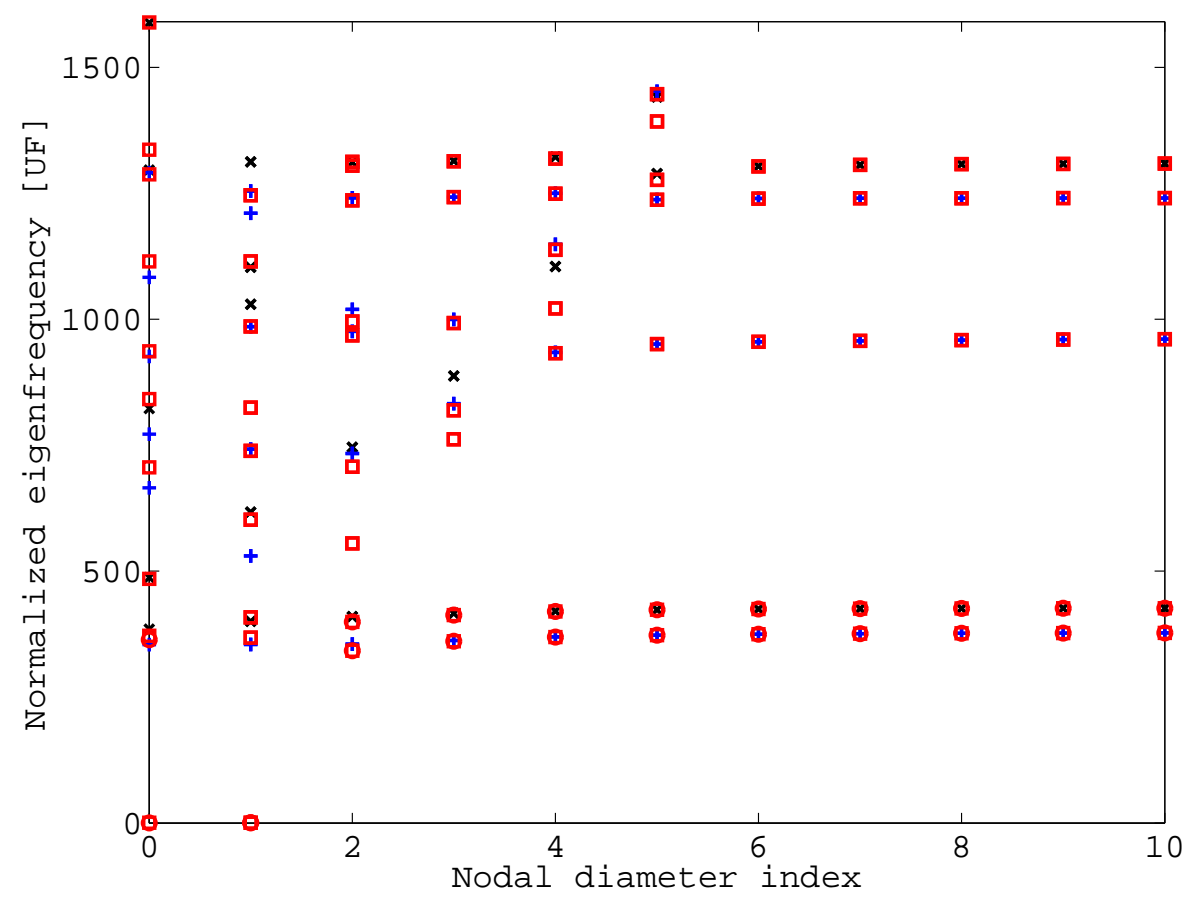

Figure 8. Influence of interstage coupling, Eigenfrequency/nodal diameter map (from 0 to $10 \mathrm{ND}$ ), stage 1 alone $\times$, stage 2 alone + , coupled stages $\square$. 


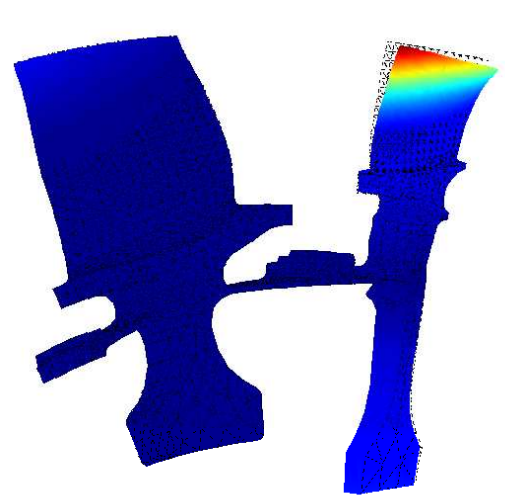

(a) $5^{\text {th }}$ mode at $0 \mathrm{ND}, 48 \mathrm{UF}$

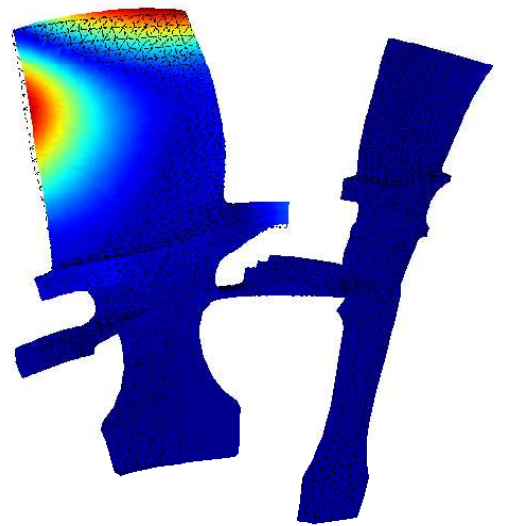

(b) $7^{\text {th }}$ mode at $9 \mathrm{ND}, 124 \mathrm{UF}$

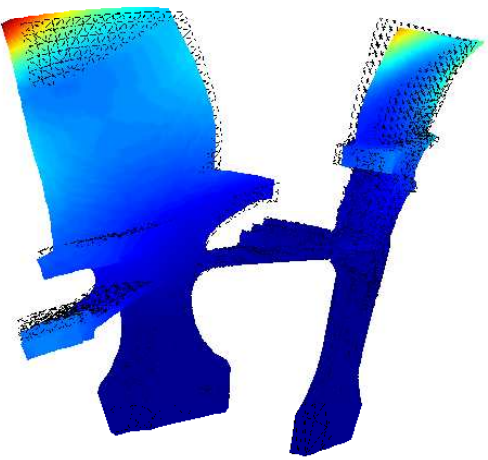

(c) $5^{\text {th }}$ modes at $3 \mathrm{ND}, 76 \mathrm{UF}$

Figure 9. Examples of modal deformed shapes

Forced response. The case of steady state response to a periodic excitation is now addressed. We consider again the two HP blisks system and we apply synchronous excitations on each stage with distinct engine orders. The excitation on stage 1 (36 sectors) is of engine order 7 and the excitation on stage 2 ( 60 sectors) is of engine order 3 . The distributions of forces are harmonic and rotating and the excitation frequency are multiple of the rotation speed and of the engine order. Figures (10), the response curves of the two stages (figure 10(a) for stage 1 and figure 10(b) for stage 2) are plotted versus the rotation speed. We compare the response of each stage alone

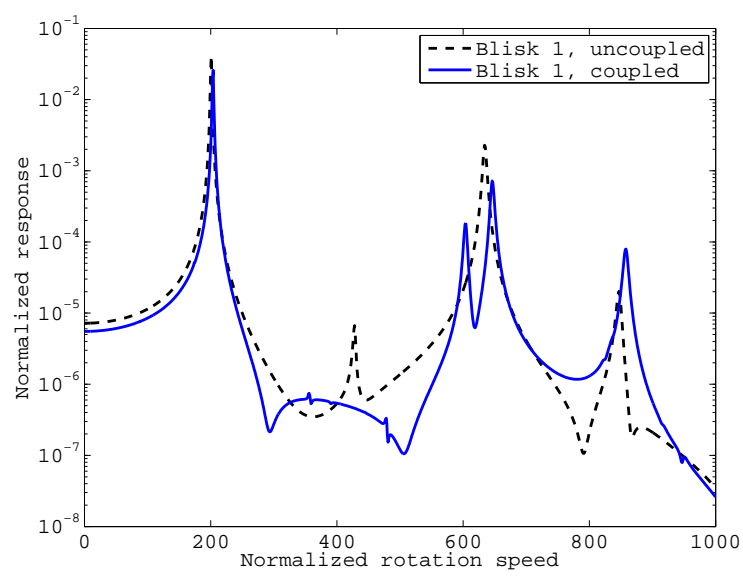

(a) Blisk 1

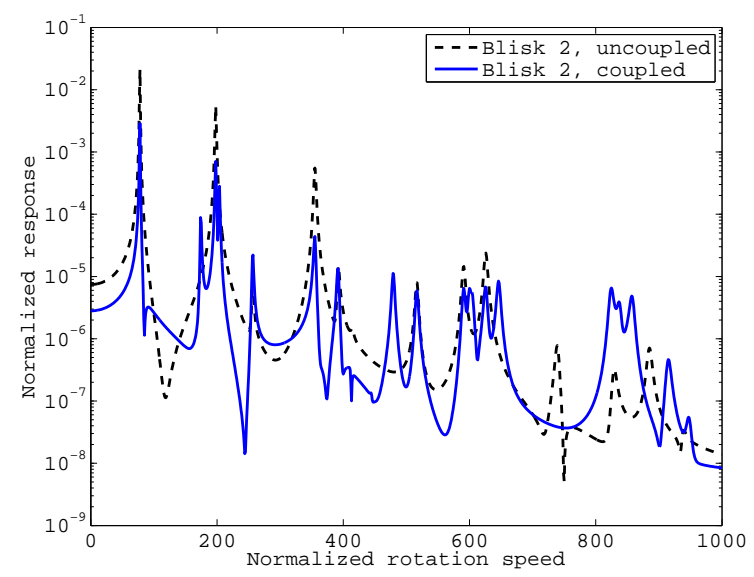

(b) Blisk 2

Figure 10. Frequency responses with and without interstage coupling

(with fixed inter-stage interface) to the two stages coupled. These response were calculated using a modal superposition and a nodal diameter superposition as explained in section 3.

The first remark would be that the multi-stage responses have a higher frequency content than the single-stage responses and that some peaks appears due to the interstage coupling. This is a major remark for the designer. In effect, as the bladed disks are usually designed by avoiding any coincidence of the modal response to the engine excitation, the possible transfer of vibratory energy between stages (adjacent or not) can, with the present method, be predicted. An other important point relates to the vibration levels which significantly differ between the 
two computations; in effect, even when the peaks location (frequency) seems to be correctly evaluated by the single stage analysis, the resonant level are generally lower in the multi-stage case. Because of the inter-stage coupling, the vibratory energy seems to spread over the two stages and this tends to reduce the vibratory levels in individual stages.

\section{Conclusions}

A new strategy to study the dynamics of multistage cyclic structures, such as turbine engine bladed disks, has been proposed. It is based on a cyclic symmetry modeling of individual stages (in their respective cyclic base) and an accurate interstage coupling in the common physical base. This method appears quite simple to carry out since the sector models of each stage are handled directly and the coupling involves few additional difficulties. The results from numerical simulations show a good accuracy of this method. Also, an industrial application highlight the importance of such calculations for the designer.

\section{Acknowledgments}

Thanks go to Snecma for its technical and financial support. This work takes place in the framework of the MAIA mechanical research and technology program sponsored by CNRS, ONERA and SAFRAN Group.

\section{References}

D. L. Thomas, "Dynamics of Rotationally Periodic Structures", International Journal for Numerical Methods in Engineering 14, 81 (1979).

J. Wildheim, "Excitation of Rotationally Circumferentially Periodic Structures", Journal of Sound and Vibration 75, 397 (1981).

A. V. Srinivasan, "Vibrations of bladed disks assemblies - A selected survey", Journal of Vibration, Acoustics, Stress, and Reliability in Design 106 (1984).

J. C. Slater, "Forced response of bladed disks assemblies - A survey", The shock and vibration digest 31, 17 (1999).

R. Bladh, M. P. Castanier, and C. Pierre, "Effects of Multistage Coupling and Disk Flexibility on Mistuned Bladed Disk Dynamics", Journal of Engineering for Gas Turbines and Power 125, 121 (2003).

R. Rzadkowski and M. Drewczynski, "Forced Vibration of Several Bladed Discs on the Shaft", in Proceedings of ASME Turbo Expo (Barcelona, Spain, 2006), GT2006-90158.

R. Bladh, M. P. Castanier, and C. Pierre, "Reduced Order Modeling Techniques for Dynamic Analysis of Mistuned Multi-Stage Turbomachinery Rotors", in Proceedings of ASME Turbo Expo (New Orleans, LA, USA, 2001), 2001- GT-0276.

S. H. Song, M. P. Castanier, and C. Pierre, "Multi-stage modeling of turbine engine rotor vibration", in Proceedings of IDETC/CIE (2005).

R. Henry, "Calcul des fréquences et modes des structures répétitives circulaires", Journal de Mécanique appliquée 4, 61 (1980). 
D. Laxalde, F. Thouverez, and J.-P. Lombard, "Dynamical Analysis of Multi-Stage Cyclic Structures", Mechanics Research Communications In press (2007a).

E. Petrov, "A method for use of cyclic symmetry properties in analysis of nonlinear multiharmonic vibrations of bladed disks", Journal of Turbomachinery 126, 175 (2004).

D. Laxalde, F. Thouverez, and J.-P. Lombard, "Vibration control for integrally bladed disks using friction ring dampers", in Proceedings of ASME Turbo Expo (Montréal, Canada, 2007b), GT2007-27087. 